Britta Amodeo, Aline Schindler, Ulrike Schacht and Hans Günther Wahl*

\title{
Calculation of indirect reference intervals of plasma lipase activity of adults from existing laboratory data based on the Reference Limit Estimator integrated in the OPUS::L information system
}

https://doi.org/10.1515/labmed-2021-0008

Received January 19, 2021; accepted March 17, 2021
Keywords: age dependent reference intervals; data mining; indirect reference limits; lipase; reference intervals.

\section{Introduction}

Laboratory results are assessed and validated on the basis of reference intervals that by definition include $95 \%$ of the results of healthy people. This is supposed to enable an efficient separation of healthy (non-diseased) and diseased subjects. Yet almost all laboratories take these values from the package inserts of in vitro diagnostics manufacturers. Although it is pointed out there that each laboratory should determine its own reference intervals for the diagnostic evaluation of patient results these data are usually used without further evaluation.

Reference intervals can be determined by direct or indirect methods. The internationally recommended direct procedure requires sample collections from a reference population with confirmed absence of particular pathological conditions $[1,2]$. Indirect methods [3, 4] select the results from a mixed population of diseased and nondiseased individuals as is usually found in medical laboratory databases. In the database not only a large number of measurement results but also other relevant information such as the date of sampling, age and sex are stored. As for each measured parameter the proportion of pathological values is small compared to the total number of all values statistical tools can be used to get the results of a probably non-diseased subpopulation. The truncated maximum likelihood (TML) model is based on the maximum likelihood estimation of the parameters of a power normal distribution for a truncated data set $[5,6]$. The truncated minimum chi-square (TMC) model is similar to the TML approach [7]. As TML and TMC do not require isolating the full value distribution from non-diseased persons,

*Corresponding author: PD Dr. Dr. Hans Günther Wahl, MBA, Medizinisches Labor Wahl, Paulmannshöherstr. 14, 58515 Lüdenscheid, Germany; and Institute of Laboratory Medicine and Pathobiochemistry, Philipps University Marburg, UKGM Marburg GmbH, Baldingerstr., 35043 Marburg, Germany,

E-mail: hg.wahl@laborwahl.de

Britta Amodeo, Aline Schindler and Ulrike Schacht, Medizinisches Labor Wahl, Lüdenscheid, Germany 
particular methods are required for estimating reference limits from truncated data.

Based on these approaches the section on Reference Limits of the German Society for Clinical Chemistry and Laboratory Medicine (DGKL) has uploaded the Reference Limit Estimator (RLE) as an R-program with MS Excelinterface on the DGKL home page [8]. In 2015 OSM AG (Essen, Germany), a commercial Laboratory Information System (LIS) provider implemented the RLE routinely in its LIS OPUS::L [9]. We present the calculation of indirect reference intervals from existing laboratory data based on RLE integrated in the OPUS::L information system using the example of Lipase.

\section{Methods}

Heparin plasma samples coming from a total of seven hospitals and from 91 doctor's offices were all analysed at the Medical Laboratory Wahl in Lüdenscheid on the ADVIA Chemistry 1800 (Siemens Healthcare Diagnostics, Tarrytown, NY, USA). No patients were excluded and no stratification of in- and out-patients were performed. In case of repeated measurements from the same patient only the first value was used.

The laboratory is accredited according to ISO 15189. The lipase assay is based on the chromogenic lipase substrate, DGGMR (1,2-odilauryl-rac-glycero-3-glutaric-acid-(6'-methylresorufin) ester). The reference interval provided by the manufacturer for lipase is $12-53 \mathrm{U} / \mathrm{L}$ for adults. Siemens states in the package insert, that as with all in vitro diagnostic assays, each laboratory should determine its own reference intervals for the diagnostic evaluation of patient results [10].

The OPUS::L "Population specific Reference Limits" based on the Reference Limit Estimator were used as previously described [5, 7, 8, 11]. The user starts the program as part of the standard LIS by choosing the measurand e.g., lipase. Once the time period of data collection and additional variables such as age group and sex are established, ambulatory or hospitalized patients from different sets of departments can be defined as subgroups. Before the estimated distribution can be calculated one has to define the type of pathological values: High $(\mathrm{H})$ is used in case of only increased values being pathological and Low (L) in case of only decreased values being pathological. Both-sides (B) is used when both increased and decreased levels are expected equally. After editing all these variables, the calculation can be started. It includes sex and age stratification, detecting drift effects during the time of data collection with drift effects being tested for significant deviations.

\section{Results}

We present the calculation of indirect reference intervals from existing laboratory data based on the Reference Limit Estimator integrated in the OPUS::L information system using the example of lipase. A total of 29.841 results (all = female and male) were included. There was no clinically significant drift in the results during the data collection within three years as shown for the 25th (green),
Drift effects during the time of data collection

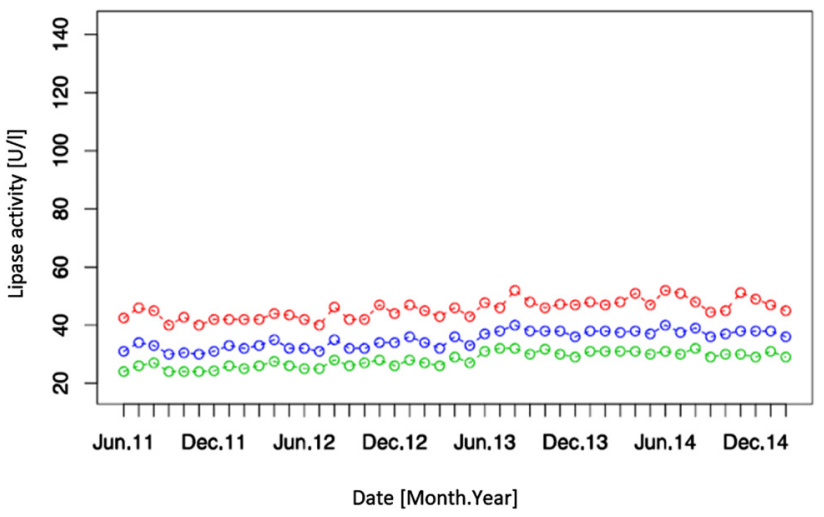

Figure 1: Time drift during data collection $(n=29.841$, all): 25 th (green), 50th (blue) and 75th (red) percentile; months.

Age Dependency

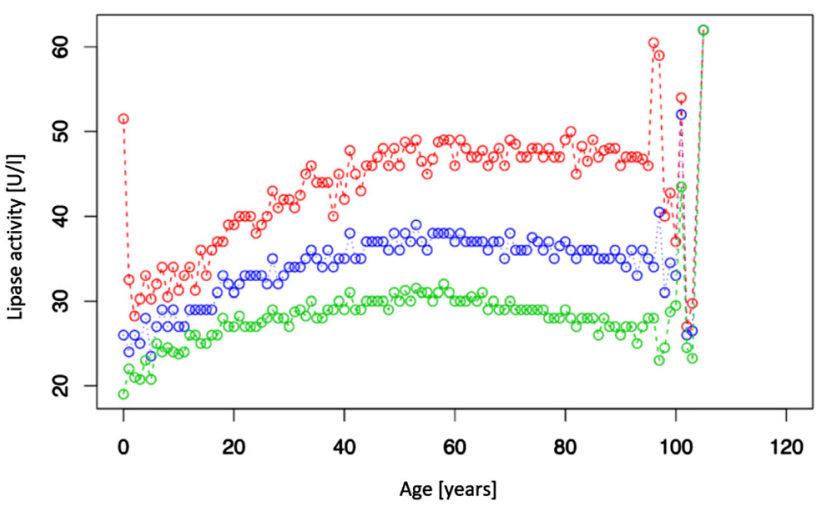

Figure 2: Age dependency ( $n=29.841$, all): 25th (green), 50th (blue) and 75th (red) percentile.

50th (blue) and 75th (red) percentile in Figure 1. The 75th percentile was between 41 and $48 \mathrm{U} / \mathrm{L}$ in the first half of the time period and between 44 and $49 \mathrm{U} / \mathrm{L}$ in the second half. A greater drift was seen for the 25 th percentile with results between 25 and $30 \mathrm{U} / \mathrm{L}$ in the first half of the time period and between 29 and $34 \mathrm{U} / \mathrm{L}$ in the second half.

Age dependencies were found for both males and females as shown for the 25th (green), 50th (blue) and 75th (red) percentile in Figure $2(\mathrm{n}=29.841)$. The extreme values at both ends of the graphs are probably due to the extremely small number of cases. Reference limits were calculated for different age groups with no relevant differences between women and men (Table 1). As an example, for an estimated distribution Figure 3 shows the curves for non-pathological, pathological values and whole data for a total number of 23.359 results from both women and men (age group 20-80 years). At the end of each calculation a summary is displayed online in the laboratory's LIS screen. 
Table 1: Upper reference limits calculated for different age groups.

\begin{tabular}{|c|c|c|c|c|}
\hline \multicolumn{6}{|c|}{ Lipase (U/I) upper reference limit } \\
\hline Age group & Male & Female & All & $\mathbf{n}$ \\
\hline $0-20$ & 41 & 41 & 41 & 1.694 \\
\hline $20-40$ & 55 & 53 & 54 & 4.203 \\
\hline $40-60$ & 62 & 62 & 62 & 7.996 \\
\hline $60-80$ & 66 & 62 & 64 & 11.160 \\
\hline$>80$ & 73 & 70 & 70 & 4.788 \\
\hline $20-80$ & 62 & 58 & 60 & 23.359 \\
\hline $0-101$ & 62 & 59 & 59 & 29.841 \\
\hline
\end{tabular}

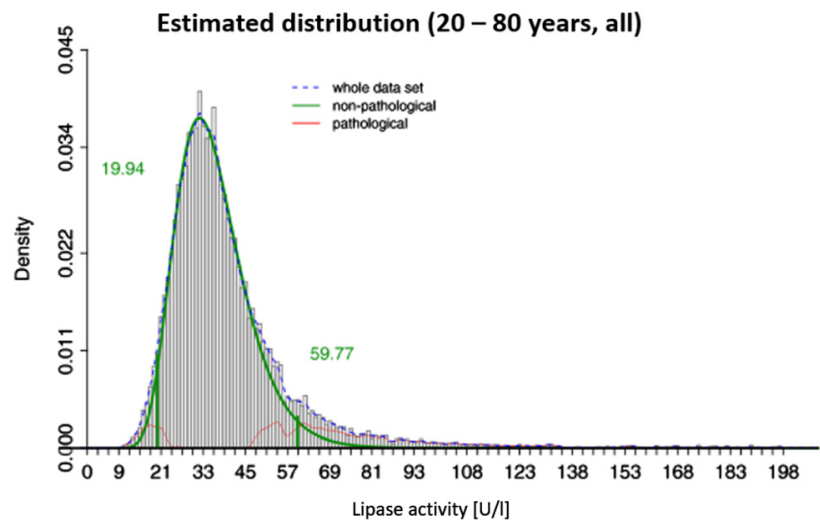

Figure 3: Estimated distribution for non-pathological values (green curve), pathological values (red) and whole data (blue). Green lines with given numbers indicate 2.5 th and 97.5 th percentile of the estimated distribution for non-pathological values (RL). $(n=23.359$, female and male, $20-80$ years old).

With the new established reference limits, we changed the manufacturer's original reference interval for lipase 12$53 \mathrm{U} / \mathrm{L}$ (adults, both women and men) to age dependent upper reference limits (uRLs) of $<60 \mathrm{U} / \mathrm{L}$ ( $20-80$ years) and $<70 \mathrm{U} / \mathrm{L}$ ( $>80$ years). In case of the age group $<20(\mathrm{n}=1.694$ years) we found an $\mathrm{URL}$ of $41 \mathrm{U} / \mathrm{L}$.

\section{Discussion}

The most prominent advantage of this OPUS::L "Population specific Reference Limits" based on the DGKL Reference Limit Estimator is the fact that it can be used online with the laboratory database. And it can be repeated very conveniently as often as required.

In many cases the RLE produces reliable results, but in some cases, especially if the data distribution is far away from normal, one has to evaluate the calculated reference limits very carefully before applying them to the routine values. Problems also can come up with the size of the specific laboratory and the test frequencies of the desired parameter. A low frequency can be overcome by a longer data collection period as long as no drift is detected. Theoretical one could combine the data from different laboratories if there are no significant differences in the data sets of the parameter under investigation. It also might be necessary in some cases to distinguish between ambulant and hospitalised patients and even further exclude patients from particular wards as dialysis, gynaecology or intensive care units.

All the estimated distributions for lipase were calculated with the pathological values option set to high, as almost all the pathological lipase values found will be increased values. Switching the option to "Both-sides" did not change the results significantly e.g., in the age group 20-80 years the $\mathrm{uRL}$ dropped from 59.8 to $59.0 \mathrm{U} / \mathrm{L}$ and the IRL raised from 19.9 to $20.2 \mathrm{U} / \mathrm{L}$. In contrast changing the option from high to low with most of the pathological values supposed to be decreased the uRL raised from 59.8 to $68.0 \mathrm{U} / \mathrm{L}$ and the $1 R L$ dropped from 19.9 to $16.9 \mathrm{U} / \mathrm{L}$ in the age group 20-80 years. So, care has to be taken to choose the right pathological values option between high and low.

Looking at the age dependent lipase reference intervals especially in the age group under 20 years it would be desirable to use this tool for a more "continuous" age dependent reference limit based on a continuous function. With the given age dependency and the small sample size in this age group the estimated uRLs should be used carefully.

In case of our newly defined lipase uRLs for adults we actually improved not only our reporting but even gained more satisfaction from our family doctors: they quite often were confronted with marginally increased lipase values in the past that needed to be controlled. With the new just barely higher reference limits these problems dropped significantly.

Research funding: None declared.

Author contributions: All authors have accepted responsibility for the entire content of this manuscript and approved its submission.

Competing interests: Authors state no conflict of interest. Informed consent: Informed consent was obtained from all individuals included in this study.

Ethical approval: Research involving human subjects complied with all relevant national regulations, institutional policies and is in accordance with the tenets of the Helsinki Declaration (as revised in 2013), and has been approved by the authors' Institutional Review Board.

\section{References}

1. Solberg HE. Approved recommendation (1987) on the theory of reference values. J Clin Chem Clin Biochem 1987;25:645-56. 
2. CLSI/IFCC. Defining, establishing, and verifying reference intervals in the clinical laboratory; approved guideline - third edition. CLSI document C28-P3. Wayne, PA: Clinical and Laboratory Standards Institute; 2008, vol 28:1-50 pp.

3. Jones GRD, Haeckel R, Loh TP, Sikaris K, Streichert TH, Katayev A, et al. Indirect methods for reference interval determination - review and recommendations. Clin Chem Lab Med 2019;57:20-9.

4. Farrell CJL, Nguyen L. Indirect reference intervals: harnessing the power of stored laboratory data. Clin Biochem Rev 2019;40: 99-111.

5. Arzideh F, Brandhorst G, Gurr E, Hinsch W, Hoff T, Roggenbuck L, et al. An improved indirect approach for determining reference limits from intra-laboratory data bases exemplified by concentrations of electrolytes. J Lab Med 2009;33:52-66.

6. Arzideh F, Wosniok W, Gurr E, Hinsch W, Schumann G, Weinstock $\mathrm{N}$, et al. A plea for intra-laboratory reference limits. Part 2. A bimodal retrospective concept for determining reference limits from intra-laboratory databases demonstrated by catalytic activity concentrations of enzymes. Clin Chem Lab Med 2007;45:1043-57.
7. Wosniok W, Haeckel R. A new estimation of reference intervals: truncated minimum chi-square (TMC) approach. Clin Chem Lab Med 2019;57:1933-47.

8. German Society of Clinical Chemistry and Laboratory Medicine. Decision limits/guideline values. Available from: https://www. dgkl.de/en/activities/sections/entscheidungsgrenzenrichtwerte/ [Accessed 15 Jan 2021].

9. https://www.osm-ag.de/fileadmin/user_upload/Produktblatt_ OpusL\%20Populationsspezifische\%20Referenzintervalle.pdf [Assessed 15 Jan 2021].

10. Siemens ADVIA Chemistry XPT Package insert Lipase (LIP) 10699649_EN Rev. H. München: Global Siemens Headquarters; 2020-02.

11. Haeckel R, Wosniok W, Torge A, Junker R, Bertram A, Krebs A, et al. Age and sex dependent reference intervals for random plasma/ serum glucose concentrations related to different sampling devices and determined by an indirect procedure with data mining. Urgent plea for studying the diagnostic efficiency of various concepts proposed to improve the pre-examination phase for determining blood glucose concentrations. J Lab Med 2021;45:95-101. 